\title{
Las redes de investigación en el marco de la revolución industrial 4.0 y la Cuarta Transformación
}

Research Networks Within the Framework of the Industry 4.0 and the "Fourth Transformation"

Redes de pesquisa no âmbito da Revolução Industrial 4.0 e da Quarta Transformação

Rosa Amalia Gómez Ortíz

Instituto Politécnico Nacional, Escuela Superior de Comercio y Administración, México

ragomez100@hotmail.com https://orcid.org/0000-0002-2082-1696

Alma Arcelia González Lozano

Instituto Politécnico Nacional, Escuela Superior de Comercio y Administración, México

almaglezloz@gmail.com https://orcid.org/0000-0002-4840-0631

Patricia Ruth Ávila Gómez

Instituto Politécnico Nacional, Escuela Superior de Comercio y Administración, México ruthavilagomez@gmail.com https://orcid.org/0000-0001-7096-6992 


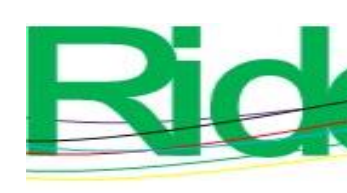

Revista Iberoamericana para la Investigación y el Desarrollo Educativo ISSN $2007-7467$

\section{Resumen}

Teniendo como telón de fondo tanto la revolución industrial 4.0 como la Cuarta Transformación, el objetivo del presente trabajo fue determinar los retos que enfrentan los coordinadores de redes de investigación en las organizaciones educativas en México. La investigación surgió ante la necesidad de crear nuevas redes de este tipo en el Instituto Politécnico Nacional (IPN). Se analizaron las características relacionadas con la innovación y la generación de nuevo conocimiento de la revolución industrial 4.0 y la Cuarta Transformación, las cuales tienen algunas similitudes aun cuando sus diferencias son más que patentes. En medio de estas dos corrientes, y ante la necesidad resolver problemas de gran envergadura, las redes de investigación son una herramienta imprescindible. El método fue inductivo-deductivo, con un estudio diagnóstico que contó con la aplicación de una guía de entrevista bajo la modalidad de debate. La muestra fue de 16 expertos. La información se manejó mediante el software Atlas.ti, con análisis reflexivo y de frecuencias. Entre los retos identificados se encuentran la falta de conocimiento del coordinador cuando se inicia una red sobre la variedad de investigadores y sobre las orientaciones respecto a un mismo tema, así como retos de comunicación y de organización. Como parte de las conclusiones, se aporta nuevo conocimiento teórico y propuestas para establecer políticas de acción para la creación y operación de redes de investigación institucionales; propuestas que pueden ayudar a mejorar la gestión y dinámica de las redes de investigadores formales e informales.

Palabras clave: cooperación en investigación, interdisciplinariedad, redes de investigación.

\section{Abstract}

In the context of both the Industry 4.0 and the "Fourth Transformation", the objective of this work was to determine the challenges faced by the coordinators of research networks in Mexican educational organizations. The research arose out of the need to create new networks of this type at the Instituto Politécnico Nacional (IPN). The characteristics related to innovation and the generation of new knowledge of the Industry 4.0 and the Fourth Transformation were analyzed, which have some similarities even when their differences are more than evident. In the midst of these two currents, and faced with the need to solve largescale problems, research networks are an essential tool. The method was inductive-deductive, with a diagnostic study that included the application of an interview guide in the form of 


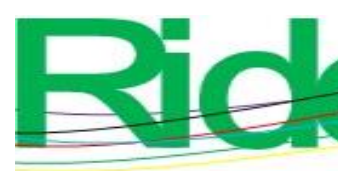

Revista Iberoamericana para la
Investigación y el Desarrollo Educativo
ISSN $2007-7467$

debate. The sample was of 16 experts. The information was managed using Atlas.ti software, with reflective and frequency analysis. Among the challenges identified are the lack of knowledge of the coordinator when starting a network on the variety of researchers and on the guidelines on the same topic, as well as communication and organizational challenges. As part of the conclusions, new theoretical knowledge and proposals are provided to establish action policies for the creation and operation of institutional research networks; proposals that can help improve the management and dynamics of networks of formal and informal researchers.

Keywords: cooperation in research, interdisciplinary, research networks.

\section{Resumo}

No contexto da Revolução Industrial 4.0 e da Quarta Transformação, o objetivo deste trabalho foi determinar os desafios enfrentados pelos coordenadores de redes de pesquisa em organizações educacionais no México. A pesquisa surgiu da necessidade de criar novas redes desse tipo no Instituto Politécnico Nacional (IPN). Foram analisadas as características relacionadas à inovação e à geração de novos conhecimentos da revolução industrial 4.0 e da Quarta Transformação, que apresentam algumas semelhanças mesmo quando suas diferenças são mais do que evidentes. No meio dessas duas correntes, e diante da necessidade de resolver problemas de larga escala, as redes de pesquisa são uma ferramenta essencial. O método foi indutivo-dedutivo, com um estudo diagnóstico que incluiu a aplicação de um guia de entrevistas na forma de debate. A amostra foi de 16 especialistas. As informações foram gerenciadas no software Atlas.ti, com análise reflexiva e de frequência. Entre os desafios identificados estão o desconhecimento do coordenador ao iniciar uma rede sobre a variedade de pesquisadores e as orientações sobre o mesmo tópico, bem como os desafios de comunicação e organização. Como parte das conclusões, novos conhecimentos e propostas teóricas são fornecidos para estabelecer políticas de ação para a criação e operação de redes institucionais de pesquisa; Propostas que podem ajudar a melhorar a gestão e dinâmica de redes de pesquisadores formais e informais.

Palavras-chave: cooperação em pesquisa, interdisciplinaridade, redes de pesquisa. Fecha Recepción: Febrero 2020

Fecha Aceptación: Agosto 2020 


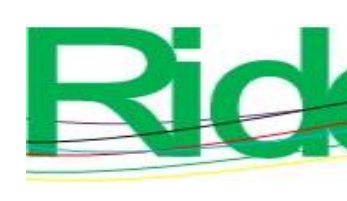

Revista Iberoamericana para la Investigación y el Desarrollo Educativo ISSN 2007-7467

\section{Introducción}

\section{La Cuarta Transformación}

La llamada Cuarta Transformación se fundamenta en tres movimientos armados importantes en la historia de México: la Independencia, la Reforma y la Revolución (Fuentes, 2018), los cuales, en su momento, cambiaron la estructura del Gobierno, así como la forma de hacer política en México, todos ellos a través de la guerra. Algunos de estos cambios tuvieron poco tiempo de duración, por tanto, no corresponden a ninguna innovación; otros, sin embargo, aún persisten en el año 2020, como la separación de la Iglesia y el Estado, y en el caso de la Revolución, la promulgación de la Constitución que rige actualmente a México. En efecto, se trata de repercusiones que transformaron profundamente la vida de los ciudadanos. Actualmente, se pretenden cambios profundos en el país considerando dos ejes: lo estructural y lo político.

Lo anterior se relaciona con aspectos de innovación. Por lo que, antes de continuar, vale la pena que definamos dicho término, innovación. Etimológicamente, la raíz latina de este concepto, innovo-are, significa 'hacer nuevo, renovar'; in alude a 'hacia adentro', y novus-a-um corresponde a 'nuevo'. Es decir, en sus inicios, se asocia a hacer, desde dentro, algo nuevo (Rivero, Asenjo y Martínez, 2009). En un contexto empresarial, Schumpeter (citado en Fagerberg, Martin y Andersen, 2013) indicó que la innovación consiste en realizar nuevas combinaciones con los recursos existentes. Aquí lo relevante es que corresponde a un proceso de cambio cualitativo en un momento histórico.

En el contexto anterior, la innovación se da en productos, métodos de producción, formas de abastecimiento, mercados o formas de organizar a las empresas o a las industrias, modificando las relaciones de la organización al interior y con su entorno.

Por tanto, la Cuarta Transformación, con fundamento en estos procesos de cambio, percibe al país como una empresa que pretende cambiar las relaciones entre los diversos actores. Sin embargo, para realizar una innovación de cualquier tipo, se requiere de planeación, organización, control y evaluación (procesos que hasta el momento más que claros han sido confusos), pasos que permitan alcanzar los objetivos propuestos. Además, los cambios deberán ser permanentes en un tiempo relativamente largo y tener la posibilidad de evaluar los resultados de la innovación pretendida. En una innovación, la transformación es en espiral, lo que significa que el conocimiento existente permite ser incrementado a través 




Revista Iberoamericana para la Investigación y el Desarrollo Educativo ISSN $2007-7467$

de mejorarlo, sin descartarlo; esto es imposible cuando se pretende generar dichos cambios de manera lineal y de arriba hacia abajo, como se observa en la Cuarta Transformación.

\section{La revolución industrial $\mathbf{4 . 0}$}

La revolución industrial 4.0 se fundamenta en procesos de innovación tecnológica. Si tenemos en mente la concepción anterior de innovación, queda por abordar la de tecnología. Pier (1989, citado en Gómez, 2014) menciona que la tecnología se deriva tanto de la teoría como de la práctica, ambos puntos son prerrequisitos de un hecho tecnológico, por tanto, la tecnología es un cuerpo de conocimientos, herramientas y técnicas derivadas de la ciencia y la experiencia práctica que es usada en una firma, producción o aplicación de productos, procesos, sistemas y servicios.

Por su parte, Lara (1998) concibe a la tecnología como un conjunto de conocimientos específicos y procesos para transformar la realidad y resolver algún problema. En el caso de Gómez (2014), determina que la tecnología es cualquier objeto (herramientas, equipos, muebles o cualquier objeto que tiene dimensiones, características y con posibilidad de ser medido), proceso o procedimiento que para su diseño y elaboración requirió un conjunto de conocimiento integrados y, por tanto, la posibilidad de ser aplicados. En forma resumida y práctica, se entiende por tecnología al conjunto de conocimientos aplicados para generar una innovación. Al cobijo de las concepciones anteriores de tecnología, se desarrolla la revolución industrial 4.0

La revolución industrial 4.0 se caracteriza por la fusión entre lo físico, lo digital y lo biológico: los límites entre estos tres campos del conocimiento se rompen y se establecen nuevas conexiones: máquinas con máquinas, máquinas con productos y productos con personas. Por tanto, esta revolución se identifica con los robots, el Internet de las cosas, la manufactura aditiva, big data, análisis de macrodatos, computación en la nube, simulación de entornos virtuales, inteligencia artificial, ciberseguridad y realidad aumentada, lo cual tiene implicaciones en los diversos sectores y redes sociales (Schwab, 2016, citado en Basco, Beliz, Coatz y Garnero, 2018).

Un indicador sustancial de los cambios sociales, las formas de relación y comunicación, las formas de consumo y hasta de visión de la vida, derivado de las diferentes revoluciones industriales, es que dichos cambios no se impusieron, no fueron planeados, se derivaron de una forma natural por el uso de la tecnología y de su transformación a través de 


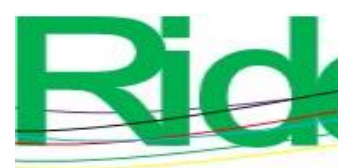

Revista Iberoamericana para la
Investigación y el Desarrollo Educativo
ISSN $2007-7467$

y la revolución industrial 4.0 estos elementos tienen propósitos diferentes. La primera busca el cambio estructural, cambiar las formas de organización que inciden en los procesos de gestión; y la revolución industrial 4.0 orienta la educación y la investigación hacia la conjunción de las diversas áreas del conocimiento con el campo del desarrollo tecnológico: digital, robots, el Internet de las cosas, la manufactura aditiva, big data, análisis de macrodatos, computación en la nube, entre otros, sin embargo, los objetivos que se pretenden se encuentran en el vaivén de las condiciones que en el tiempo se presenten.

De esta manera, los investigadores en el trabajo en red encuentran retos de contexto, de política pública en el campo de la ciencia y la tecnología, la cual, en ocasiones, es rebasada por la velocidad del cambio tecnológico, social y ambiental, además de los problemas de organización en la dinámica interna de las redes, todas estas situaciones y otras, como decíamos, deben ser enfrentadas de manera conjunta por los miembros de las redes.

\section{Importancia y conceptualización de las redes}

El trabajo en equipo y la colaboración se han convertido en los vehículos para la integración de conocimientos, esfuerzos y capacidades para los procesos de investigación. Esto ha requerido ir más allá de los límites disciplinarios, institucionales y geográficos para el estudio y solución de estos problemas por parte de la comunidad científica (Bermeo, De los Reyes y Bonavia, 2009). Entre las propiedades de las redes que han sido más analizadas en los estudios de administración o que siguen la perspectiva del capital social, se encuentran la centralidad de la red, la densidad de esta, su tamaño y la presencia de agujeros estructurales en ellas.

La centralidad es la propiedad que identifica a los nodos con mayor número de vínculos dentro de una red (Wasserman y Faust, 1994). Los actores más centrales pueden tener una posición de ventaja respecto a los demás en tanto que tienen más alternativas para satisfacer sus necesidades, más posibilidades de acceso a recursos y se considera que son menos dependientes (Hanneman y Riddle, 2005). A los nodos más centrales en la estructura de una red también se les denomina nodos estrella, ya que por su alto grado de relación sobresalen de los demás

Luo (2005) relaciona la centralidad de la red del grupo con aspectos del liderazgo al considerar que una red centralizada puede presentar un liderazgo fuerte, y que es posible que 


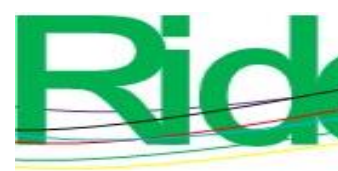

Revista Iberoamericana para la Investigación y el Desarrollo Educativo ISSN $2007-7467$

exista mayor control de las actividades del grupo y mayor organización, aunque también una red muy centralizada puede limitar la libertad de acción de los integrantes del grupo.

\section{Concepción del término redes}

Newman (2000), en su artículo sobre la estructura de las redes, define red social como una colección de personas, cada una de las cuales está familiarizado con algún subconjunto (entendiendo subconjunto como los elementos que, por alguna característica o conocimiento específico, conforman un grupo que forma parte del grupo más amplio). Sin embargo, Newman (2000), al hablar de redes de colaboración, puntualiza que su estudio se ubica en el campo de las redes sociales, y lo confirma en el siguiente párrafo:

Las redes sociales han sido objeto tanto de carácter empírico como de estudios teóricos en las ciencias sociales durante al menos 50 años, en parte debido al interés inherente a los patrones de interacción humana, también porque su estructura tiene importantes implicaciones para la difusión de la información y de la enfermedad. Está claro, por ejemplo, que la variación promedio del número de individuos implicados, también llamado grado medio de la red, podría influir sustancialmente en la propagación de un rumor, una moda, una broma o la fiesta de este año (pp. 406-407).

Aun así, vale la pena destacar su definición, ya que, a pesar de ser desarrollada para las redes sociales, se puede considerar idónea para cualquier tipo de red, incluyendo las redes creadas con propósitos de investigación.

Uno de los aspectos que otorgan importancia a las redes de investigación es la posibilidad de publicaciones en coautoría, ya que, como lo menciona Adams (2012), las nuevas redes regionales están reforzando la competencia y capacidad de investigación en las economías emergente. Una forma para enfrentar los desafíos de las instituciones educativas y centros de investigación es la colaboración vista desde una perspectiva pública más amplia. Por ejemplo, la transferencia de conocimientos puede darse de mejor manera al estar en condiciones de colaboración y las publicaciones de artículos en coautoría son citados con mayor frecuencia.

La coautoría derivada del trabajo de investigación en red ha incrementado el número de coautores en los artículos. En los años 80, raro era observar más de tres o cuatro autores. 


\begin{tabular}{|l|l|} 
Tabla 1. Clasificación de las redes \\
\hline \multicolumn{1}{|c|}{ Clasificación } & \multicolumn{1}{c|}{$\begin{array}{c}\text { Revista Iberacterísticas } \\
\text { Investigación y el Desarrollo Ed }\end{array}$} \\
\hline Por su alcance & $\begin{array}{l}\text { Institucional, nacional, regional } \\
\text { o internacional }\end{array}$ \\
\hline Propósito u objetivo & $\begin{array}{l}\text { Redes de información y } \\
\text { comunicación, redes } \\
\text { académicas, redes temáticas, } \\
\text { redes de investigación, redes de } \\
\text { innovación y redes de servicios } \\
\text { tecnológicos }\end{array}$ \\
\hline $\begin{array}{l}\text { Por la naturaleza de su } \\
\text { asociación }\end{array}$ & \begin{tabular}{l} 
Formales e informales \\
\hline
\end{tabular}
\end{tabular}

Fuente: Elaboración propia

Conforme a la clasificación de la tabla 1, la presente investigación corresponde a una de alcance institucional; por su objetivo, es de investigación; por su naturaleza, es tanto formal como informal.

En las redes de investigación, los miembros colaboran para realizar investigaciones de mayor envergadura y complejidad, por lo que se requiere el trabajo interdisciplinario. Las redes de investigación, o también llamadas por algunos más redes de conocimiento, consideran la participación tanto de los investigadores como de los usuarios de la investigación. Se complementan las capacidades y hay un reparto equitativo de tareas. Su importancia radica en que han transformado los modos de producción del conocimiento y del desarrollo de la tecnología; al revalorizar la colaboración, posibilita la interdisciplinariedad. Abordan problemas complejos y favorecen la mejora de capacidades investigativas.

\section{Colaboración-cooperación}

En un mundo globalizado, la importancia de la investigación para resolver problemas comunes en diversos países y distintas comunidades adquiere mayor relevancia. Y cuando se trata de problemáticas de alto grado de complejidad, el trabajo colaborativo adquiere todavía mayor importancia. Según Schlattmann (2017), existen numerosas razones por las que la colaboración es importante en la ciencia y destaca: 1) los científicos más destacados buscan trabajar con los colegas más destacados en su campo; 2) los enfoques de investigación interdisciplinarios determinan procesos de colaboración, a través de centros de investigación interdisciplinarios, redes institucionales o centros de excelencia, y 3) existe una mayor apreciación de la investigación interdisciplinaria por parte de las organizaciones de financiamiento. 


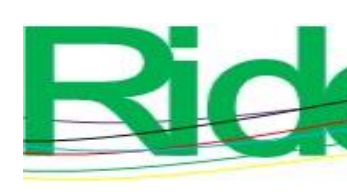

Revista Iberoamericana para la Investigación y el Desarrollo Educativo ISSN 2007 - 7467

Pero las posiciones y perspectivas teóricas de lo que se entiende por cooperación y colaboración son encontradas. Por ejemplo, Schneider (2006, citado en Gómez, 2019) determinó una diferencia entre ambos términos. A la cooperación la definió como "un proceso en el que todos trabajan hombro con hombro en una misma dirección y con una misma finalidad. Se trata de un trabajo en pleno acuerdo entre las partes, más que un trabajo en competencia" (p. 7). La colaboración es diferente, corresponde a "un proceso en el que los individuos trabajan juntos en una compleja intersección de fines comunes, aunque desde actitudes de competencia e intereses diversos" (Schneider, 2006, citado en Gómez, 2019, p. 7). Por tanto, mientras la cooperación es un trabajo colectivo desde el consenso, la colaboración, para Schneider (2006, citado en Gómez, 2019), es un trabajo colectivo en conflicto, donde se confía en los otros mientras más se busca el logro de los propios intereses y donde la pluralidad de posiciones no es reducida.

El término colaboración en el mundo se ha manejado de manera indistinta con otros términos como cooperación y alianzas. La colaboración ocurre a nivel de individuos o instituciones. En el caso de la colaboración entre organizaciones, incluye relaciones recíprocas bien definidas para lograr metas comunes, y la colaboración entre individuos se define como un proceso para compartir la creación de algo, donde los sujetos interactúan para crear y compartir el conocimiento o para su desarrollo. En este sentido, la colaboración es un proceso para compartir el conocimiento, pero también para lograr metas comunes (Bermeo et al., 2009).

\section{Comunicación}

Existe aspectos que no pueden dejarse de lado en un trabajo de investigación colaborativa a través de las redes, si se considera como una interacción social: la cultura, el idioma y las características particulares que están incrustadas en una actividad de investigación científica, sobre todo cuando el trabajo es multidisciplinario y en cada área de conocimiento existe un lenguaje técnico especial para denominar conceptos similares pero con significados diferentes o significados similares con términos diferentes. En este marco, los investigadores prefieren trabajar con quienes utilizan su mismo lenguaje, entre pares de la misma área, o de aquellos que no dominan el idioma inglés, los cuales serán menos solicitados para colaborar. 


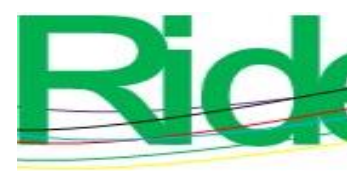

Revista Iberoamericana para la Investigación y el Desarrollo Educativo ISSN 2007-7467

\section{Instituto Politécnico Nacional y sus redes de investigación}

El Instituto Politécnico Nacional (IPN) se creó en 1936. Es la primera institución a nivel nacional en el campo de la ciencia y la tecnología y la segunda por la cantidad de matriculados. El número de sus programas asciende a 249, los cuales se agrupan en tres áreas: ingeniería y ciencias físico-matemáticas con 156 programas, ciencias médico-biológicas con 59 programas y ciencias sociales con 34. La oferta integra los niveles educativos de bachillerato, licenciatura, maestría y doctorado. En el periodo 2019-2020, en el sistema escolarizado estuvieron 185358 alumnos y en el no escolarizado 5895, de tal manera que la matrícula ascendió a la cantidad de 191253 alumnos. Su distribución es a lo largo de la república mexicana, con una concentración importante en Ciudad de México (IPN, 2020a). De los programas que oferta el nivel posgrado, 105 se encuentran en el Programa Nacional de Posgrados de Calidad (PNPC). Un alto porcentaje de sus egresados son de alto nivel y reconocidos a nivel internacional. Actualmente, dos de los mejores investigadores del área de robótica y del área de la salud, junto con otros investigadores del país, representarán a México en los trabajos para los protocolos y la vacuna contra la COVID-19 (IPN, 21 de mayo de 2020).

\section{Redes de investigación en el IPN}

Las redes de investigación y posgrado del IPN se crearon a partir del 30 de noviembre de 2006 como órganos de asesoría, consulta, apoyo y coordinación de esta casa de estudios. Su finalidad es la de "promover la formación de recursos humanos de excelencia académica y profesional, así como la generación de conocimientos científicos de vanguardia y su transformación en aplicaciones útiles a la sociedad en dicha materia" (Secretaría de Investigación y Posgrado [SIP] y Coordinación de Redes de Investigación y Posgrado [Coriyp], 2019, párr. 4).

Los lineamientos para la admisión de profesores a alguna de las redes de investigación, entre otros aspectos, son: 1) tener nombramiento de profesor de 40 horas, de tiempo completo; 2) en los últimos tres años, haber publicado trabajos relacionados con la especialidad de la red a la cual solicita su incorporación; 3) presentar su currículo del Consejo Nacional de Ciencia y Tecnología (Conacyt) — esto porque debe ser parte del Sistema Nacional de Investigadores (SNI) — actualizado con productividad de los últimos tres años; 4) haber participado durante los últimos tres años en proyectos de investigación formales en 


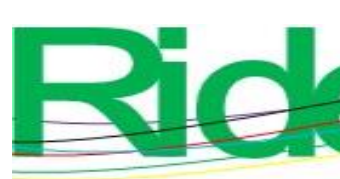

Revista Iberoamericana para la Investigación y el Desarrollo Educativo ISSN 2007 - 7467

tópicos de la red a la cual se solicita la incorporación, en caso de las redes de expertos (en estos casos no se requiere ser miembro del SNI) deberán contar con evidencia de trabajos profesionales como asesor, consultor o participación en la elaboración de normas en las áreas de telecomunicaciones o robótica y mecatrónica o haber participado en proyectos de investigación formales con financiamiento interno, externo o en proyectos vinculados o contar con publicaciones en los últimos tres años en las áreas ya mencionadas; 5) aceptar cumplir las obligaciones de los miembros de las redes de investigación y posgrado, descritos en el acuerdo de creación de estas, y 6) no pertenecer a más de dos redes de investigación o expertos del IPN.

Como se puede observar, un investigador, previamente a su ingreso a una red de investigación, debe contar con investigaciones personales o individuales para integrarse a investigar a la red que le interesa. En la tabla 2 se muestra el número de miembros, número de publicaciones, proyectos multidisciplinarios en los que participaron, número de patentes sometidas para registro, esto en el año 2016. El número de publicaciones es mínimo debido a que los resultados de las investigaciones son de tipo tecnológico, por lo que esto se refleja en el número de patentes sometidas a registro.

Tabla 2. Características de las redes

\begin{tabular}{|l|c|c|c|c|}
\hline \multicolumn{1}{|c|}{ Redes } & Miembros & Publicaciones & $\begin{array}{c}\text { Proyectos } \\
\text { multidisciplinarios }\end{array}$ & Patentes \\
\hline Biotecnología & 139 & 2 & 37 & 28 \\
\hline Medio ambiente & 110 & 3 & 28 & 4 \\
\hline $\begin{array}{l}\text { Nanociencia y micro } \\
\text { nanotecnología }\end{array}$ & 94 & 9 & 34 & 9 \\
\hline Computación & 74 & 0 & 8 & 2 \\
\hline Energía & 66 & 1 & 3 & 0 \\
\hline Desarrollo económico & 72 & 0 & 4 & 2 \\
\hline $\begin{array}{l}\text { Telecomunicaciones } \\
\text { (expertos) }\end{array}$ & 37 & 0 & 7 & 16 \\
\hline Salud & 59 & 0 & 3 & $\mathbf{7 3}$ \\
\hline $\begin{array}{l}\text { Expertos en robótica y } \\
\text { mecatrónica }\end{array}$ & 63 & 0 & $\mathbf{1 3 5}$ & \\
\hline Total & $\mathbf{7 1 4}$ & $\mathbf{1 5}$ & & 2 \\
\hline
\end{tabular}

Fuente: Necoechea (2018)

En el marco de la revolución industrial 4.0, la cual marca lineamientos de desarrollo tecnológico, y la orientación política de la Cuarta Transformación, cuyo propósito es el cambio de las estructuras organizacionales y políticas, y tomando en cuenta la necesidad de 


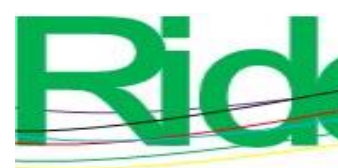

Revista Iberoamericana para la
Investigación y el Desarrollo Educativo
ISSN $2007-7467$

trabajar en red para resolver problemas de gran envergadura, las preguntas de investigación que guiaron la presente fueron las siguientes:

- ¿Qué tipo de problemas enfrentaron los coordinadores de redes de investigación en la creación y organización de una red?

- ¿ ¿Qué elementos de la revolución industrial 4.0 y de la Cuarta Transformación favorecerían la creación y organización de redes de investigación?

\section{Método}

Se utilizó el método inductivo-deductivo. Incluyó un análisis reflexivo entorno a la información obtenida a través de un debate denominado Experiencias en la conformación, operación y permanencia de redes de investigación. En total, en el debate participaron 16 personas, entre las que se encontraron tanto excoordinadores como coordinadores de las redes. El impacto que se tuvo fue considerable, pues asistieron como oyentes 1200 personas entre directivos, investigadores y estudiantes de licenciatura, maestría y doctorado.

Los temas que orientaron el debate fueron: retos que se enfrentaron para la organización y estructuración de la red; problemas que afrontaron en la operación de la red: administrativos, de organización de los investigadores, resistencias, integración de grupos; estrategias que se implementaron para generar cohesión en la red, e influencia de la tecnología para la operación de la red.

La información se analizó a través del software Atlas.ti. Además, se hizo uso del análisis de frecuencias de categorías identificadas, así como un análisis reflexivo que permitió alcanzar los siguiente objetivos: a) identificar los principales retos de operación e integración de una red de investigación y b) detectar los resultados de investigación en las diferentes redes.

\section{Resultados}

Fueron ocho áreas que presentaron mayor dificultad, según los excoordinadores y coordinadores de las redes, para la organización y operación de estas: 1) Desconocimiento de los coordinadores sobre quién investiga sobre el tema de la red; 2) Inseguridad por parte de los miembros para mantener su productividad personal en la red; 3) Organización; 4) Comunicación; 5) Apoyos; 6) Normatividad; 7) Tamaño de la red, y 8) Trabajo en equipo. 

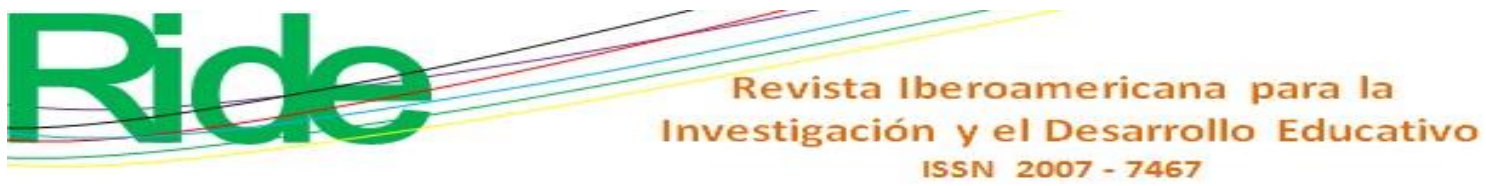

En la tabla 3 y en la figura 2 se muestran los resultados más significativos. En la figura 1 se muestran los retos de mayor frecuencia.

\section{Los problemas que enfrentaron los coordinadores en la creación y organización de una red}

Hay que tener presente que el IPN es una institución a nivel nacional y tiene campus en toda la república mexicana, por ello es complicado determinar dónde y quiénes trabajan sobre las líneas de la red, y también sobre cómo se trabaja en ella.

Para el trabajo con otras redes, el reto fue buscar miembros activos de otras de estas que les interesara participar en los proyectos de nanotecnología y computación, los cuales ya estaban tocando temas de salud en sus trabajos. Algunos aspectos más con los que se enfrentó fue la disposición de tiempo suficiente, ya que generalmente los investigadores realizan diversas actividades como investigación, actividades académicas y de índole personal, pero, sobre todo, se requería el deseo de participar.

Figura 1. Problemas identificados



Nota: Los retos identificados y de los cuales no se encontró algún estudio relacionado con ellos fueron: normatividad, apoyos económicos, inseguridad e ignorancia;

y los de mayor frecuencia y que afecta a todas las redes es la comunicación y la organización.

Fuente: Elaboración propia 

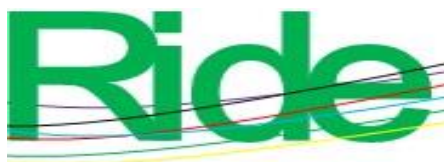

Además de lo ya mencionado, también hay que tomar en cuenta que cuando se inician las redes poco se sabe de la expertise de cada miembro. Al haber varias especialidades, el reto es que los integrantes se sumen al trabajo colaborativo.

Otro de los aspectos que se caracterizaron como reto fue la comunicación a través de la tecnología. Si bien este reto se manejó en términos de comunicación, también está relacionado con la infraestructura, ya que si no se cuenta con una plataforma informática rápida, extensa y eficaz que pueda contener y procesar la información que se genera, el trabajo se complica. En la tabla 3 se destacan los principales retos enfrentados por las redes de investigación. 


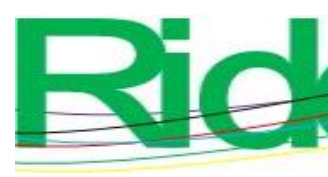

Revista Iberoamericana para la Investigación y el Desarrollo Educativo ISSN 2007 - 7467

Tabla 3. Principales retos enfrentados por las redes de investigación

\begin{tabular}{|c|c|}
\hline Reto & Características \\
\hline \multirow[t]{4}{*}{ Desconocimiento } & $\begin{array}{l}\text { En la red de salud, el reto fue integrar miembros vigentes que } \\
\text { les interesara participar en proyectos nanotecnología y } \\
\text { computación en las redes. }\end{array}$ \\
\hline & $\begin{array}{l}\text { Desconocimiento de quiénes y dónde, en el mismo IPN, hacían } \\
\text { investigación sobre el tema de biotecnología (hay que } \\
\text { considerar que el instituto tiene centros de investigación en todo } \\
\text { el país). }\end{array}$ \\
\hline & Poco se sabía del expertise de cada miembro. \\
\hline & $\begin{array}{l}\text { Cada investigador tiene expertise diferente, por lo que el reto es } \\
\text { introducirlos al trabajo colaborativo. }\end{array}$ \\
\hline \multirow[t]{10}{*}{ Retos de comunicación } & $\begin{array}{l}\text { Los investigadores cambian frecuentemente los correos } \\
\text { electrónicos, lo que genera problemas serios para la } \\
\text { comunicación y la socialización de la información. }\end{array}$ \\
\hline & $\begin{array}{l}\text { Dificultad para poner en contacto a los investigadores de los } \\
\text { diferentes centros. }\end{array}$ \\
\hline & $\begin{array}{l}\text { Comunicación a través de la tecnología. Si no se tiene una } \\
\text { plataforma informática rápida, extensa y eficaz que pueda } \\
\text { contener información, el trabajo se complica. }\end{array}$ \\
\hline & $\begin{array}{l}\text { Falta mayor comunicación, debido a que requieren mejores } \\
\text { canales de comunicación. }\end{array}$ \\
\hline & $\begin{array}{l}\text { Conjuntar y considerar que todos somos expertos en el área y } \\
\text { que el otro no siempre tiene la razón, aun cuando se trabaje en } \\
\text { lo mismo. }\end{array}$ \\
\hline & $\begin{array}{l}\text { Falta comunicación más efectiva entre los investigadores de los } \\
\text { centros o de las instituciones de educación superior con las } \\
\text { empresas, ya que los tiempos se manejan de manera diferente. } \\
\text { El tiempo de una investigación para un investigador puede durar } \\
\text { ocho años; para un empresario debe durar meses y lleva el sello } \\
\text { de pesos. En el caso del Gobierno, es necesario que se den } \\
\text { cambios y el impacto, en medida de lo posible, debe darse } \\
\text { dentro de un periodo gubernamental. }\end{array}$ \\
\hline & Falta de comunicación entre miembros. \\
\hline & $\begin{array}{l}\text { La traducción de las diferentes formas de expresión técnica } \\
\text { entre los miembros que pertenecen a diferentes áreas del } \\
\text { conocimiento, así como a las metodologías que utilizan. }\end{array}$ \\
\hline & $\begin{array}{l}\text { No se sabe, en muchas ocasiones, en cuántos proyectos de } \\
\text { investigación participan para solucionar problemas nacionales, } \\
\text { se tiene que hacer una búsqueda exhaustiva. }\end{array}$ \\
\hline & $\begin{array}{l}\text { La complejidad de los seres humanos puede impedir la } \\
\text { comunicación. }\end{array}$ \\
\hline Retos de organización & Se muestran en la figura 2. \\
\hline
\end{tabular}

Fuente: Elaboración propia 


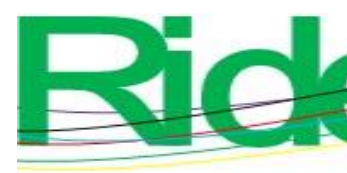

Revista Iberoamericana para la
Investigación y el Desarrollo Educativo
ISSN $2007-7467$

Algunos de los investigadores establecen redes de investigación con investigadores de otras instituciones; el problema es la dificultad para que estos investigadores informen con quién lo hacen y si es a nivel nacional o internacional. Por lo que se desconoce qué investigadores y en cuántos proyectos participan para solucionar problemas nacionales. Este problema se extiende a la dificultad para obtener información del Conacyt.

Un reto que pareciera simple, pero que en una red de estas características se convierte en algo complejo, es la forma de expresión técnica que se genera en las diferentes áreas del conocimiento. El trabajo en red incluye la interdisciplinariedad. Esto genera limitaciones y, en el menor de los casos, restricciones para avanzar en el trabajo. Otro desafío son las metodologías que utilizan dependiendo del área de conocimiento.

Una amenaza para la vinculación es la falta de comunicación efectiva entre los investigadores de los centros o de las instituciones de educación superior con las empresas, debido fundamentalmente a que los tiempos de ambos sectores son diferentes. El tiempo de una investigación para un investigador puede durar ocho años; para un empresario debe durar meses, por el sello de pesos que esto conlleva.

\section{La organización como reto en una red}

En este punto se presentan problemas que parecieran personales, pero que se integraron a este rubro ya que las actividades familiares se traslapan con las profesionales, más cuando algún coordinador de red es al mismo tiempo director de escuela o de algún centro de investigación o sección de posgrado.

Como se mencionó con anterioridad, a diferencia de una red de investigación, una red de expertos es más flexible en sus requisitos. La red de robótica se conformó como una de expertos pues cada nivel de expertise en este campo es relevante, no obstante, el problema de organización que se presenta es el de aglutinar sus esfuerzos en pocas líneas de investigación, ya que cada miembro tiene su propia línea.

Este siguiente problema lo enfrentan en todas las instituciones, por ello se considera comprensible, ya que todo individuo tiene una vida personal que necesita compaginar con la actividad laboral y de investigación, en el caso de los investigadores; así, la pregunta que se hacen es: ¿cómo se organiza una persona cuando trabaja hasta 12 horas diarias? Es complicado atender alumnos, dirigir tesis, dar clases en licenciatura y posgrado, ver dónde se publican los resultados de investigación y cómo y qué se escribe. 

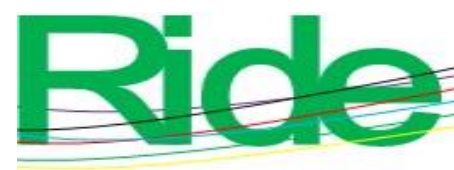

Revista Iberoamericana para la Investigación y el Desarrollo Educativo ISSN $2007-7467$

Entre la carga de trabajo que cada investigador realiza, se encuentra, aunado a la labor de desarrollar investigaciones e impartir clase a los grupos asignados semestralmente, la de cumplir con los trámites administrativos que solicita la SIP como parte de su función. Los investigadores siempre están en la búsqueda y en la entrega de documentos para solicitar los estímulos por productividad a los que tiene derecho, además de todas las actividades antes mencionadas.

Entre los problemas de organización a los que se enfrentaron los coordinadores, también se encuentran los siguientes: falta de motivación por parte de los miembros para el trabajo en red; aplicación de los elementos del proceso de organización, esto se reflejó en el momento en que, a nivel central, se dieron cambios de administración en la Coriyp, lo que afectó la organización al interior de las redes, por ejemplo, de los 120 miembros en la red de energía, solo quedaron 20; hace falta mayor integración, sobre todo en la red de energía; la carga de trabajo de los coordinadores de una red debe realizarse sin personal de apoyo, ya que, generalmente, además de ser coordinadores de red, son funcionarios o profesores, o realizan las tres funciones al mismo tiempo. Sin duda se requiere mayor enlace entre el representante de nodo y los miembros de ese nodo. 


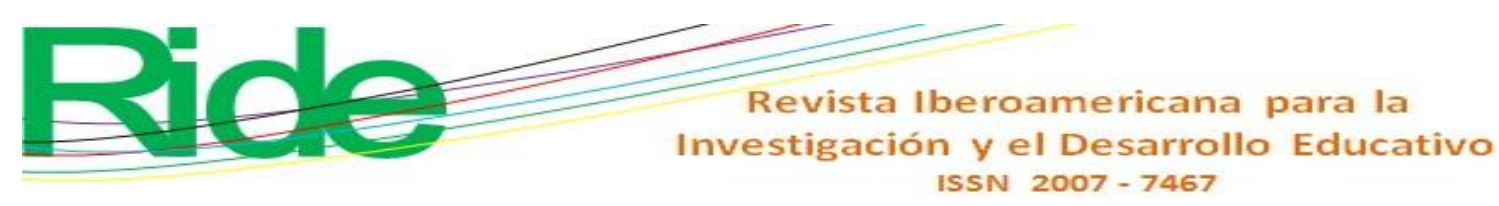

Figura 2. Retos de organización

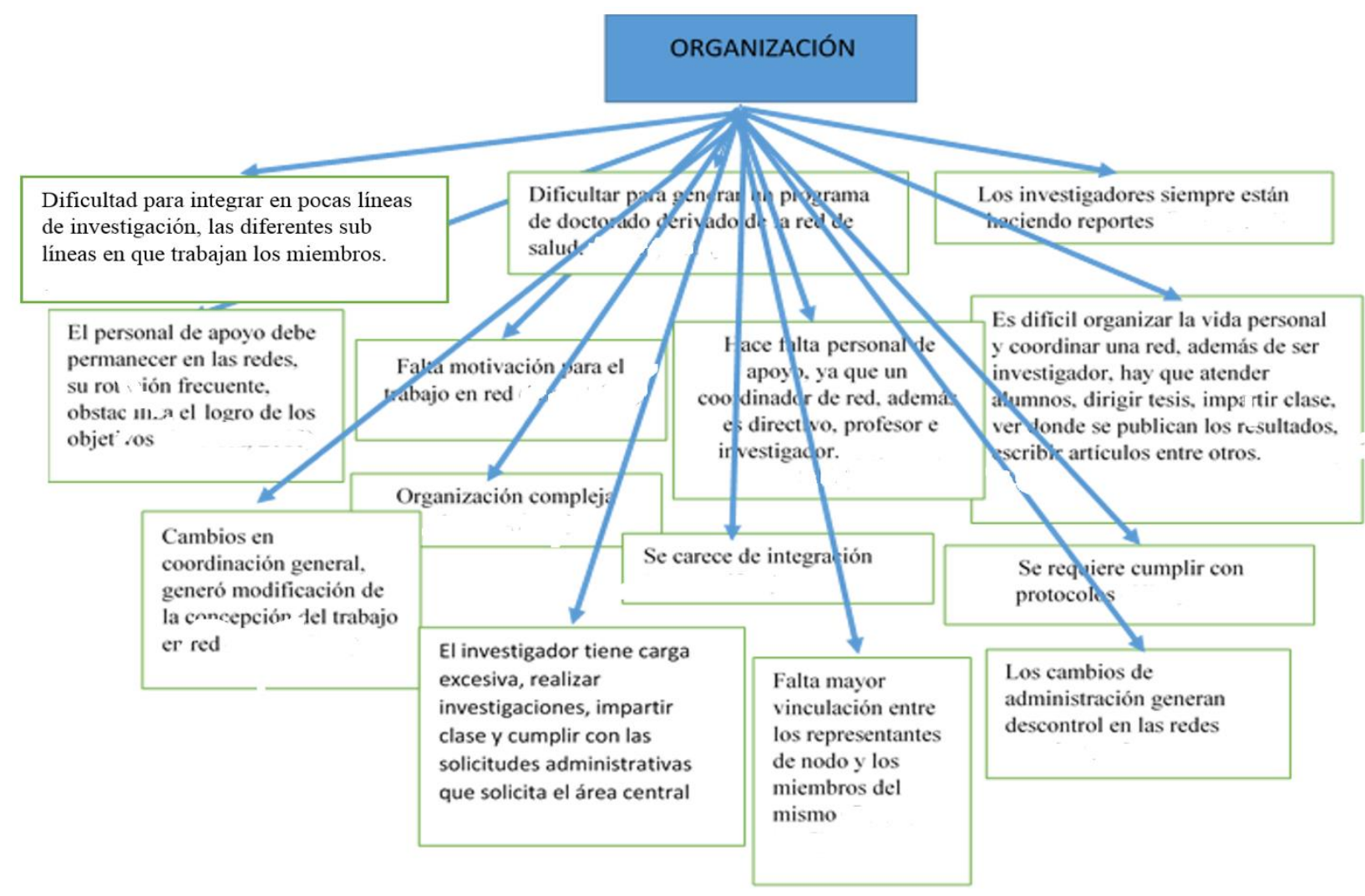

Fuente: Elaboración propia

Otro de los problemas a los que se enfrentan los coordinadores es la frecuencia con que se da la rotación de personal de apoyo. En las redes donde sí existe, este personal es importante para lograr los objetivos, por lo que es necesaria su permanencia como soporte.

\section{Discusión}

Si bien tanto a la revolución industrial 4.0 como a la Cuarta Transformación se les puede ubicar en el campo de la innovación, sin embargo, existen diversos aspectos de esta última que podrían limitar el desarrollo de las redes de investigación, entre los que se encuentran la falta de una planeación estratégica, elementos de seguimiento, evaluación del impacto, pero sobre todo, la forma de colaboración, ya que las redes trabajan en colaboración horizontal y en la Cuarta Transformación las decisiones se toman de arriba hacia abajo.

En el caso de la revolución industrial 4.0, cuyo origen está naturalmente vinculado con el desarrollo de la ciencia y la tecnología, con trabajo colaborativo horizontal por parte de expertos investigadores que facilitaron el incremento de dicho conocimiento, en forma dinámica y en espiral, cuya permanencia en el tiempo corresponde a condiciones naturales y 


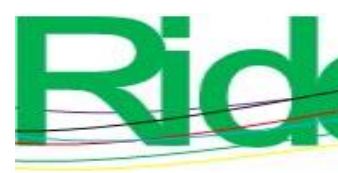

Revista Iberoamericana para la Investigación y el Desarrollo Educativo ISSN 2007 - 7467

depende de la velocidad con la que los conocimientos se transforman, teniendo en cuenta todo ello, este escenario, por supuesto, facilita el trabajo en red para la investigación científica y tecnológica direccionada hacia la solución de problemas de gran envergadura.

La congruencia entre una y otra radica en que las políticas públicas, dependiendo en cómo se determinen, pero sobre todo en cómo se gestionen, impactarán a ambas en mayor o menor medida, al trabajo de investigación y especialmente a la gestión de los coordinadores de las redes. El mayor impacto de la Cuarta Transformación será en la organización y el cambio de estructuras en instituciones y de la revolución industrial 4.0, en los apoyos económicos y de infraestructura para el desarrollo de la investigación.

En este trabajo se aporta evidencia empírica de los retos y la dificultad que enfrentan los coordinadores de redes para saber el número de investigadores que abordan un mismo tema, a nivel nacional, y sus diferentes aristas. Entre los estudios que se han llevado a cabo sobre las redes, se encuentran como tópicos centrales la colaboración y la cooperación, así como las barreras de comunicación entre los miembros de las redes.

\section{Conclusiones}

Se concluye que los retos a los que se enfrentaron los investigadores en el proceso para crear y coordinar las redes de investigación en el IPN fueron: 1) falta de información por parte de los coordinadores de las redes sobre quiénes investigan sobre el tema que les interesa a estas en los diferentes campus del IPN; 2) inseguridad de los investigadores por mantener su productividad personal por el hecho de participar en la red; 3) problemas de organización; 4) problemas de comunicación; 5) falta de apoyos económicos; 6) normatividad en construcción; 7) tamaño de la red, y 8) trabajo en equipo.

Los retos de mayor incidencia corresponden a las categorías de organización en la red y en las formas de comunicación, esto incluye instrumentos, capacidades y cobertura de la red.

Las redes de investigación en el IPN son de tipo institucional, y se requiere mayor enlace entre la Coriyp con los coordinadores de cada red y con sus miembros, lo cual induce a la centralización por parte de la autoridad y flexibilidad en la operación. 


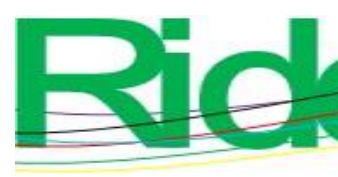

Revista Iberoamericana para la
Investigación y el Desarrollo Educativo
ISSN $2007-7467$

Una conclusión relevante fue el identificar que, para el manejo de una red de investigación, se requiere de un coordinador que sea reconocido ampliamente por los investigadores, ya que, en una red donde todos los miembros son investigadores nacionales, se genera una dinámica de egos y, por lo tanto, la dificultad para los coordinadores se incrementa al coordinar a líderes en su área de conocimiento y que son expertos en su campo.

Respecto al marco político y estructural de la Cuarta Transformación y de la revolución industrial 4.0 como desarrollo y transformación de la tecnología, se concluyó que ambas corrientes han incidido en la generación de cambios de tipo social, económico y político.

\section{Propuestas}

La coordinación de redes debería tener la posición de una dirección dentro de la SIP, ya que el número de redes de investigación, así como las redes de expertos y las redes que se van creando, requieren de atención de acuerdo con sus propósitos, estructura y dinámicas, lo que facilitaría la creación de una red de redes conforme las necesidades nacionales e institucionales

Generar infraestructura física y humana en las diferentes sedes donde se encuentre el coordinador de cada una de las redes. El funcionamiento de la red en procesos de gestión dependerá del trabajo colaborativo de docentes e investigadores.

Es necesaria la participación de las ciencias sociales en el campo de las ciencias médicas, biológicas y en el campo de todas las redes de la ingeniería. Una visión administrativa siempre es necesaria, no como elementos técnicos, sino como investigadores en el campo de conocimiento de las redes.

El trabajo de temas específicos pero amplios que requieran resultados prontos; deberán trabajar en grupos de red pequeños para alcanzar un trabajo funcional.

Algunas líneas de investigación que surgen de esta investigación son: financiamiento a las redes de investigación para su operación y desarrollo; evolución de las redes; comparación entre la organización y comunicación informal de una institución y la organización comunicación informal de las redes; agrupación política interna a las redes, y liderazgos en el funcionamiento de las redes. 


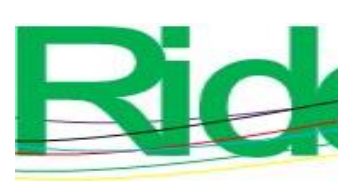

Revista Iberoamericana para la
Investigación y el Desarrollo Educativo
ISSN $2007-7467$

\section{Agradecimientos}

La presente investigación se realizó con la valiosa colaboración de diferentes personajes, entre ellos, los coordinadores y excoordinadores de las redes de investigación, quienes fueron los creadores de dichas redes y se enfrentaron a los retos tanto de organización como de creación e integración de las redes. Se agradece el apoyo del director de la Escuela Superior de Comercio y Administración, Unidad Santo Tomás, y del secretario de la SIP del IPN, y la colaboración en los procesos de organización y apoyo durante la realización del debate a los diferentes jóvenes becarios, que participaron con entusiasmo.

\section{Referencias}

Adams J. (2012). The rise of research networks. Nature, 490, 335-336. Retrieved from https://www.nature.com/articles/490335a.

Bermeo, H. P., De los Reyes, E. and Bonavia, T. (2009). Dimensiones of the scientific collaboration and its contribution to the academic research groups scientific quality. Research Evaluation, 18(4), 301-311

Basco, A., Beliz, G., Coatz, D. y Garnero, P. (2018). Industria 4.0: Fabricando el futuro. Buenos Aires, Argentina: Unión Industrial Argentina / Banco Interamericano de Desarrollo / Instituto para la Integración de América Latina y el Caribe.

Bozeman, B. and Corley, E. (2004). Scientists' collaboration strategies: Implications for scientific and technical human capital. Research Policy, 33(4), 599-616. Retrieved from https://www.sciencedirect.com/science/article/abs/pii/S0048733304000162?via\%3 Dihub.

Fagerberg, J., Martin, B. R. and Andersen, E. S. (2013). Innovation Studies. Evolution and Future Challenges. New York, United States: Oxford University Press.

Fuentes, Y. (26 de noviembre de 2018). ¿Qué es la "Cuarta Transformación” que propone Andrés Manuel López Obrador para México? BBC. News | Mundo. Recuperado de https://www.bbc.com/mundo/noticias-america-latina-45712329.

Gómez, R. A. (2014). Gestión del conocimiento, tecnología e innovación. México: Limusa. Hanneman, R. A. and Riddle, M. (2005). Introduction to social network methods. Riverside, United States: University of California. Retrieved from http://faculty.ucr.edu/ hanneman/. 


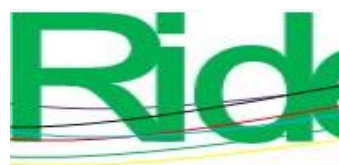

Revista Iberoamericana para la
Investigación y el Desarrollo Educativo
ISSN $2007-7467$

Instituto Politécnico Nacional [IPN]. (2020). Informe de autoevaluación 2019. México: Instituto Politécnico Nacional. Recuperado de www.ipn.mx.

Instituto Politécnico Nacional [IPN]. (21 de mayo de 2020). Participará IPN en protocolo internacional que busca desarrollar una vacuna contra COVID-19. Comunicado de prensa.

Lara, R. F. (coord.) (1998). Tecnología, conceptos, problemas y perspectivas. Actores y procesos en la innovación tecnológica. México: Siglo XXI / Universidad Nacional Autónoma de México.

Lee, S. and Bozeman, B. (2005) The impact of research collaboration on scientific productivity. Social Studies of Science, 3(5), 673-702.

Luo, J. D. (2005). Social network structure and performance of improvement teams. International Journal of Business Performance Management, 7(2), 208-223.

Necoechea, M. (2018). Fortalecimiento de las redes de investigación en el IPN. México: Dirección de Publicaciones del Instituto Politécnico Nacional.

Newman, M. E. J. (2000). Structure of scientific collaboration networks. Proceedings of the National Academy of Sciences, 98(2), 404-409. Retrieved from https://www.pnas.org/content/98/2/404.

Rivero, F., Asenjo, L. y Martínez, P. (2009). Claves para innovar en marketing y ventas. España: Wolters Kluwer. en http://es.scribd.com/doc/54192853/1/Elorigen-latino.

Schlattmann, S. (2017), Capturing the collaboration intensity of research institutions using, social network analysis. Procedia Computer Science, 106, 25-31. Retrieved from https://www.sciencedirect.com/science/article/pii/S1877050917302739?via\%3Dihu b.

Schneider, F. (2006). Collaboration: The Dark Side of the Multitude. Research Policy, 33(2004), 599-616.

Sebastián, J. (2000), Redes de cooperación como modelo organizativo y funcional para la I + D. Redes, 7(15), 97-111. Recuperado de https://ridaa.unq.edu.ar/bitstream/handle/20.500.11807/702/04_R2000v7n15.pdf?se quence $=1 \&$ is Allowed $=\mathrm{y}$.

Secretaría de Investigación y Posgrado [SIP] y Coordinación de Redes de Investigación y Posgrado [Coriyp]. (2019). Misión y Visión de la Coordinación de Redes de la 


\begin{tabular}{|c|c|}
\hline Rol de Contribución & Autor (es) \\
\hline Conceptualización & (Rosa Amalia Gómez, Principal. Hugo Necoechea igual) \\
\hline Metodología & (Rosa Amalia Gómez, Principal) \\
\hline Software & (Patricia Ruth Ávila) igual \\
\hline Validación & ( Rosa Amalia Gómez principal y Luis Rocha Lona, Apoyo) \\
\hline Análisis Formal & Patricia Ruth Ávila Gómez, Apoyo \\
\hline Investigación & $\begin{array}{l}\text { (Rosa Amalia Gómez, principal, Luis Rocha Lona, igual, } \\
\text { Alma Arcelia González, Apoyo, Patricia Ruth Ávila, Apoyo) }\end{array}$ \\
\hline Recursos & (Rosa Amalia Gómez Principal, Hugo Necoechea, igual) \\
\hline Curación de datos & $\begin{array}{l}\text { (Luis Rocha, principal, Alma Arcelia González, apoyo y } \\
\text { Patricia Ruth Ávila, apoyo) }\end{array}$ \\
\hline $\begin{array}{l}\text { Escritura - Preparación del } \\
\text { borrador original }\end{array}$ & $\begin{array}{l}\text { Rosa Amalia Gómez Ortiz, Principal, Alma Arcelia González, } \\
\text { igual) }\end{array}$ \\
\hline $\begin{array}{l}\text { Escritura - Revisión y } \\
\text { edición }\end{array}$ & $\begin{array}{l}\text { Rosa Amalia Gómez, principal; Alma Arcelia González, igual, } \\
\text { Patricia Ruth Ávila, apoyo) }\end{array}$ \\
\hline Visualización & (Rosa Amalia Gómez, principal) \\
\hline Supervisión & Rosa Amalia Gómez, principal (directora del proyecto) \\
\hline $\begin{array}{l}\text { Administración de } \\
\text { Proyectos }\end{array}$ & (Rosa Amalia Gómez, principal) \\
\hline Adquisición de fondos & (Rosa Amalia Gómez, principal) \\
\hline
\end{tabular}

